\title{
Isoelectric Point
}

National Cancer Institute

\section{Source}

National Cancer Institute. Isoelectric Point. NCI Thesaurus. Code C54157.

The $\mathrm{pH}$ at which a molecule in solution has no net charge and does not migrate in an electric field. 